\title{
Design of Energy Efficient Sinusoidal PWM Waveform Generator on FPGA
}

\author{
Sujeet Pandey ${ }^{1}$, Puneet Tomar ${ }^{2}$, Lubna Luxmi Dhirani ${ }^{3}$, D M Akbar Hussain ${ }^{4}$, \\ Vishal Jain ${ }^{5}$ and Nisha Pandey ${ }^{2}$ \\ ${ }^{1}$ Jain University, Bangalore, India \\ ${ }^{2,6}$ Gyancity Research Lab, Gurgaon, India \\ ${ }^{3}$ University of Limerick, Ireland \\ ${ }^{4}$ Aalborg University, Esbjerg, Denmark \\ ${ }^{5}$ Bharati Vidyapeeth's, Institute of Computer Applications and Management, India \\ welcomesujeet@gmail.com,puneettomar91@gmail.com,lubna.luxmi@ul.ie, \\ akh@et.aau.dk,vishaljain83@ymail.com,nisha@gyancity.com
}

\begin{abstract}
In this investigation, we are designing an energy efficient sinusoidal PWM Waveform Generator on a FPGA which obviously consumes less amount of power. The used power is obtained by varying ambient temperature level and then checking corresponding amount of energy consumed. There is a reduction of Leakage power in the percentage of $60.86 \%, 52.17 \%, 39.13 \%$ and $21.73 \%$ when we scaled down ambient temperature from $50(C)$ to $0,12.5,25,37.5(C)$ respectively. SPARTAN-6 family FPGA is being used to implement sinusoidal PWM waveform generator.
\end{abstract}

Keywords: Energy Efficient, Sinusoidal PWM Waveform Generator, FPGA, VHDL

\section{Introduction}

The basic idea here is to generate or synthesize a sinusoidal PWM waveform by passing a digitally generated PWM through a low pass filter that is technically, known as Direct Digital Synthesis (DDS). We have analyzed the varying power with different levels of temperature in order to develop the energy efficient sinusoidal PWM waveform generator.. In this paper, we have used four different clock frequencies $50 \mathrm{MHz}, 71.42$ $\mathrm{MHz}, 125 \mathrm{MHz}$ and $500 \mathrm{MHz}$ and four wave pulse frequencies $33.33 \mathrm{MHz}, 47.61 \mathrm{MHz}$, $83.33 \mathrm{MHz}$ and $333.33 \mathrm{MHz}$ respectively for the different level of ambient temperature level varying from $0(\mathrm{C})$ to 50 (C) with a step value of 12.5 (C). Spartan-6 family FPGA is used for simulation and monitoring of total power consumed. SPARTAN-6 family has greatest system integration abilities and some points about SPARTAN-6 are highlighted Figure 1.

Received (June 27, 2017), Review Result (September 4, 2017), Accepted (September 8, 2017) 
Three Spartan-6 which are SLICEL, SLICEM and SLICEX.

Every CLB has two slices which are arranged side by side as a part of two vertical columns.

The power consumed is almost half the power consumed by previous families.

It offers more effective and efficient look up table i.e. LUT logic.

Figure 1. Some Important Feature about Spartan6

\subsection{RTL Schematic}

RTL is acronym for Register Transfer Level. RTL of our designed sinusoidal waveform generator is shown in Figure 2.

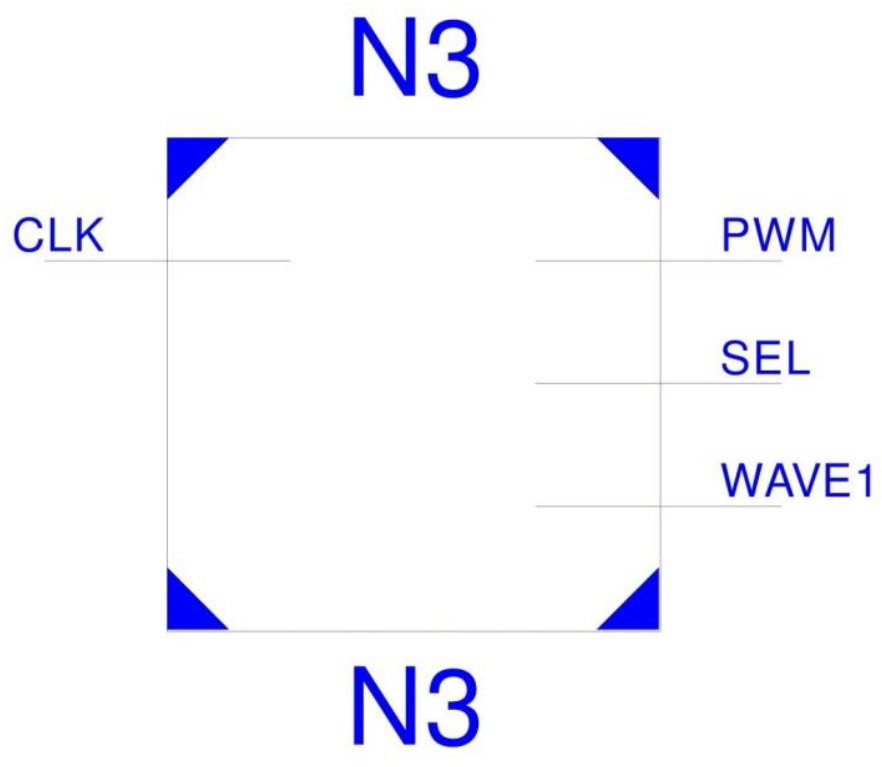

Figure 2. Basic Architecture of the RTL Schematic

As seen in the Figure 2, the RTL has one input clock port and three output ports such as PWM, SEL, and WAVE1. RTL schematics of sinusoidal PWM Waveform Generator. This block diagram define the external architecture of the N3 model which has total four port one is clock input port and others three are output ports which provides the PWM waveform. 


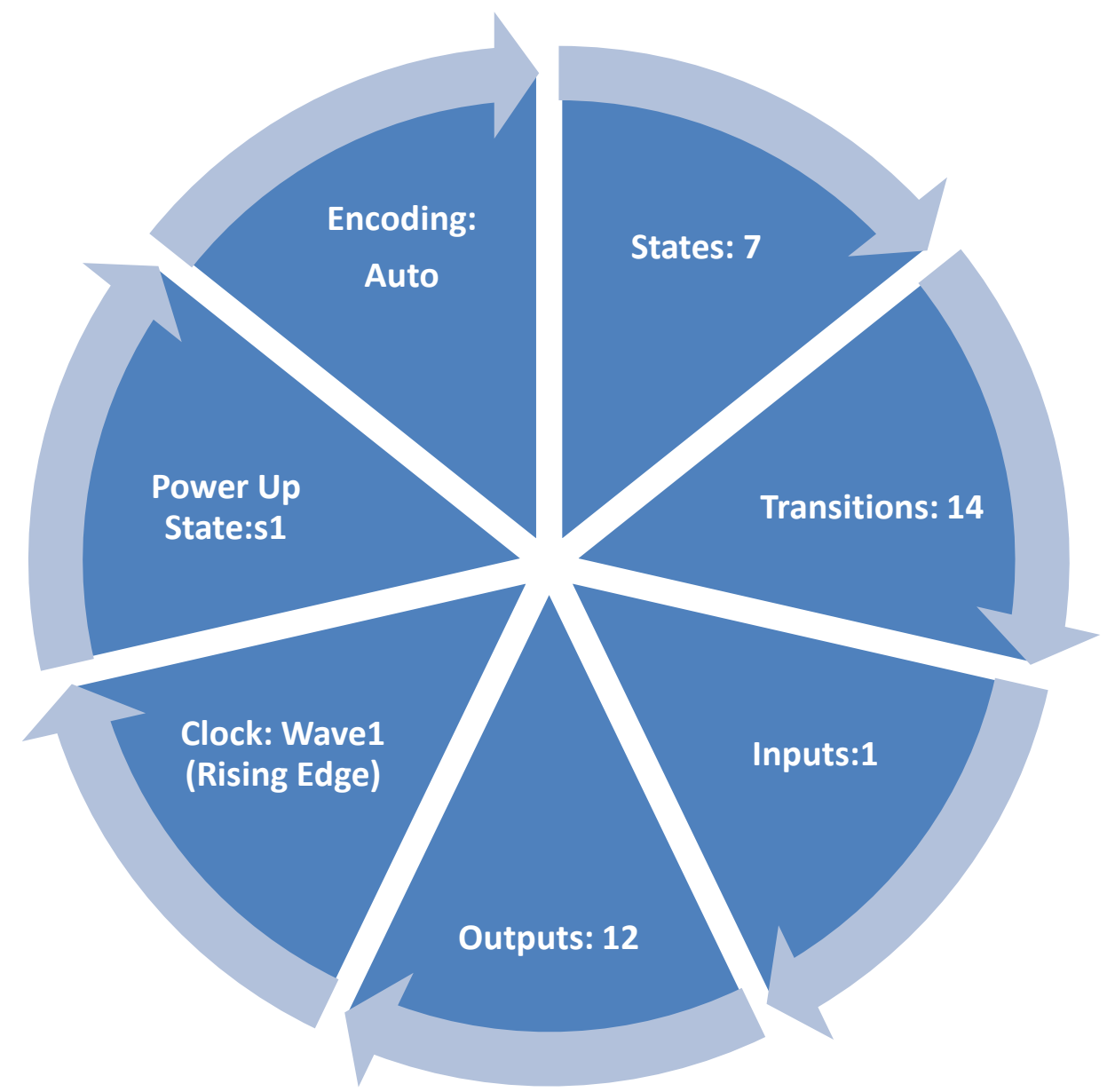

Figure 3. Initial Feature of the Synthesizing Modal N3 Design

As shown in Figure 3, N3 model has eight HDL synthesizing units for example number of States, Transitions, Inputs, Outputs are 7, 14, 1, and 12 respectively. Clocks pulse is rising edge WAVE1, power Up State is connected to state s1. Figure 4 illustrates internal architecture of RTL schematics of sinusoidal PWM waveform generator. In this type of model, we can take HDL synthesis report that contains Macro Statistics of components, which are being used in the model. This design is using 3 adders, 5 registers, 1 comparator and 1 finite state machine (FSM). 


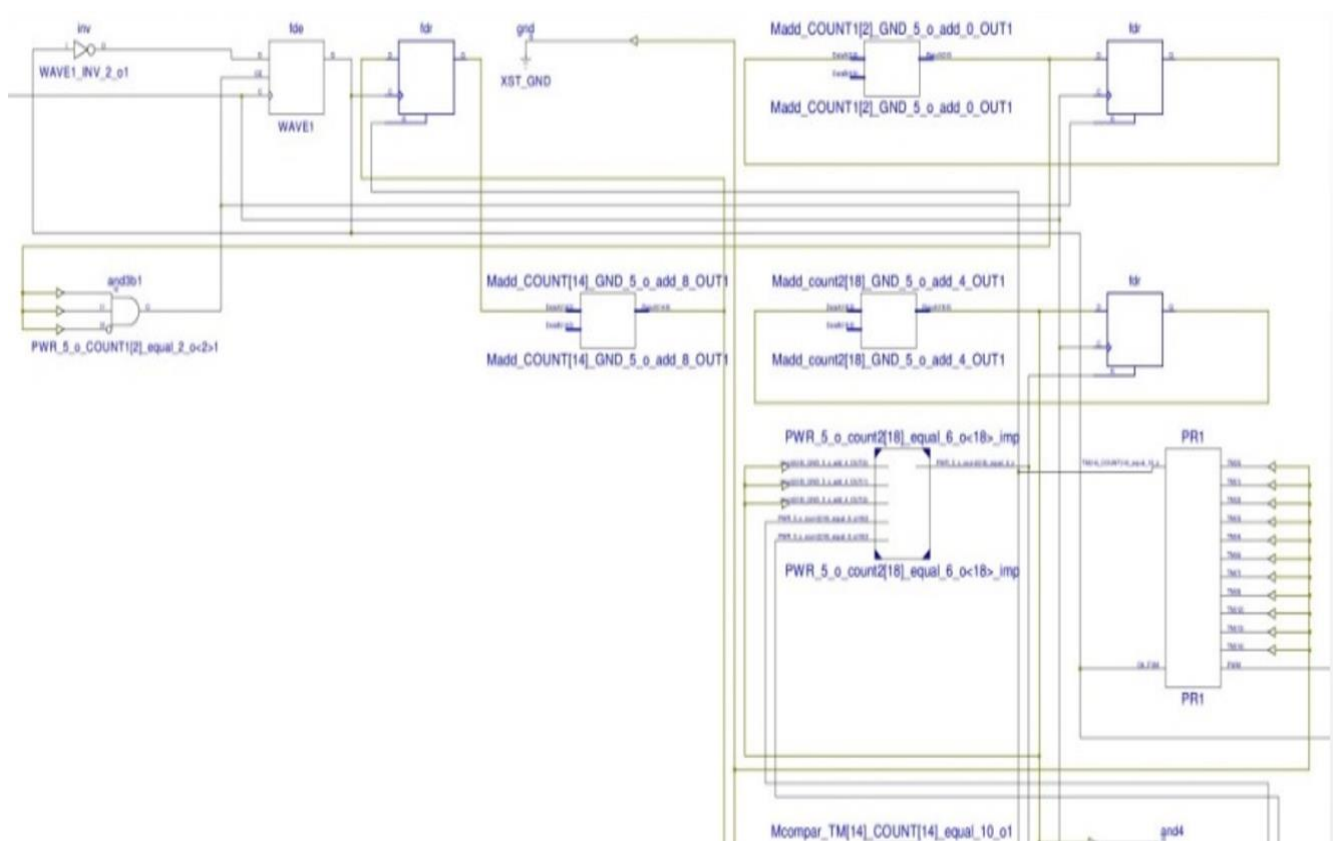

Figure 4. Internal Architecture of RTL Schematics of Sinusoidal PWM Waveform Generator (Part 1)

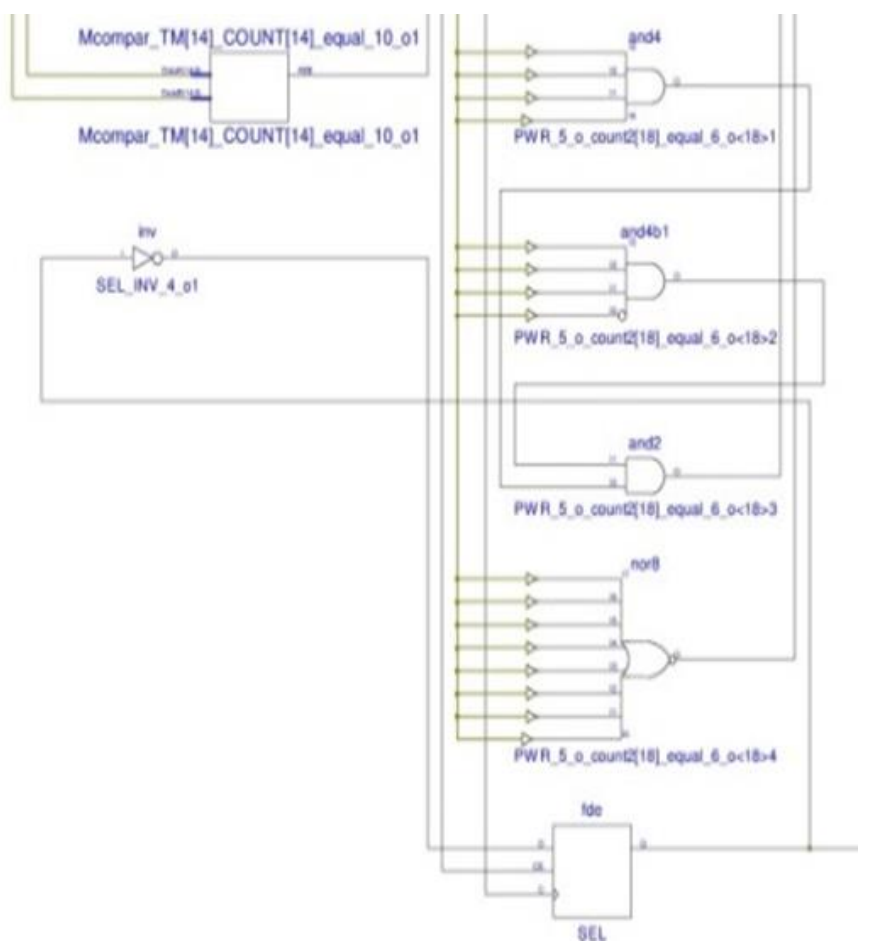

Figure 5. Internal Architecture of RTL Schematics of Sinusoidal PWM Waveform Generator (Part 2)

\subsection{Technology Schematic}

The RTL Schematic describe the technical architecture of the behavior of the model design. It has seven states and corresponding encoding bits are shown in the Figure 4 and Table 1. Technology schematic illustrates its actual implementation on FPGA. 
Table 1. State and its Corresponding Encoding Bits of Finite State Machine

\begin{tabular}{|c|c|}
\hline State & Encoding \\
\hline S1 & 000 \\
\hline S2 & 001 \\
\hline S3 & 010 \\
\hline S4 & 011 \\
\hline S5 & 100 \\
\hline S6 & 101 \\
\hline S7 & 110 \\
\hline
\end{tabular}

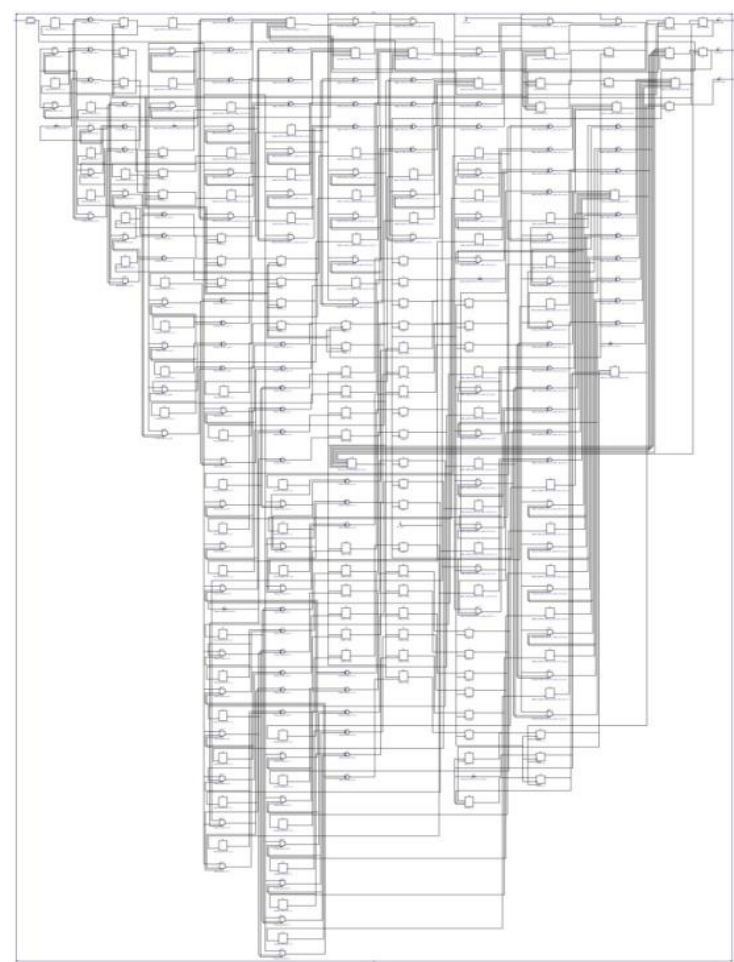

Figure 5. Technology Schematic of Sinusoidal PWM Waveform Generator

\section{Related Works}

In this reference research [1], work have been investigated and an energy efficient ALU using Mobile DDR IO standards has been designed. In our work, we are making Energy efficient PWM in place of ALU. We are using thermal scaling and frequency scaling in place of Mobile DDR IO standards. In this reference [2] researcher have designed an energy efficient Multiplier using Nikhilam Navatashcaramam Dashatah Vedic technique. Another researcher have performed power dissipation analysis of DES algorithm, implemented on a 28nm FPGA [3]. Some researcher have used thermal aware approach in an encoder design and also testing thermal stability by working on different ambient temperatures [4]. In our investigation, we are also using thermal and frequency scaling for making the design energy efficient. In this, reference [5] researchers have made energy efficient thermal aware image ALU Design on a FPGA. In this work researcher has tried to analyze the energy optimization possibility in counter design by the selection of energy efficient IO standards [6]. We are using different levels of thermal and frequency scaling in place of IO standards. In this research investigation researcher is using 11 different IO standards from HSTL and LVCMOS family to explore the 
feasibility of Vedic Multiplier in Data Encryption Algorithm, DSP, Reliable System, Multimedia and Fault Tolerant Systems [7]. In this reference work [8] researcher have made thermal efficient ALU Design by using six different members of SSTL IOs standards. Researcher in this investigation have performed power analysis of DES algorithm, which is being implemented on a 28nm FPGA using HTML (H-HSUL, TTTL, M-MOBILE_DDR, L-LVCMOS) technology [9].

\section{Results}

Table 2. Power Dissipation when Clock Period is 20ns and Period of Wave Pulse is 30 ns

\begin{tabular}{|l|l|l|l|l|l|l|}
\hline $\begin{array}{l}\text { Ambient } \\
\text { Temperature } \\
\text { (in C) }\end{array}$ & $\begin{array}{l}\text { Clocks } \\
\text { Power } \\
(\mathrm{W})\end{array}$ & $\begin{array}{l}\text { Logic } \\
\text { Power } \\
(\mathrm{W})\end{array}$ & $\begin{array}{l}\text { Signals } \\
\text { Power } \\
(\mathrm{W})\end{array}$ & $\begin{array}{l}\text { IOs } \\
\text { Power } \\
(\mathrm{W})\end{array}$ & $\begin{array}{l}\text { Leakage } \\
\text { Power } \\
(\mathrm{W})\end{array}$ & $\begin{array}{l}\text { Total } \\
\text { Power } \\
(\mathrm{W})\end{array}$ \\
\hline 0 & 0.002 & 0.000 & 0.000 & 0.008 & 0.009 & 0.019 \\
\hline 12.5 & 0.002 & 0.000 & 0.000 & 0.008 & 0.011 & 0.021 \\
\hline 25 & 0.002 & 0.000 & 0.000 & 0.008 & 0.014 & 0.024 \\
\hline 37.5 & 0.002 & 0.000 & 0.000 & 0.008 & 0.018 & 0.028 \\
\hline 50 & 0.002 & 0.000 & 0.000 & 0.008 & 0.023 & 0.033 \\
\hline
\end{tabular}

We can see from Table 2 that there is a fall of $60.86 \%, 52.17 \%, 39.13 \%$ and $21.73 \%$ in the Leakage Power as the ambient temperature is scaled down from 50 (C) to $0,12.5$, 25, 37.5 (C) Degree Celsius Respectively. The total power goes low by a percentage of $46.66 \%, 40 \%, 30 \%$, and $16.66 \%$ as the temperature level is scaled down from 50(C) to 0 , $12.5,25,37.5$ (C) respectively. Figure 6 . is displaying leakage and total power for the range of ambient temperature. There is no change in Clock Power (W) which has a constant value $0.001 \mathrm{~W}$ and IOs Power(W) with a constant value $0.006 \mathrm{~W}$ when ambient temperature is increasing from $0(\mathrm{C})$ to $50(\mathrm{C})$. Also note that there was no Logic Power (W) and Signal Power (W) consumed by the circuit within the range of our ambient temperature scale.

\section{Power Dissipation}

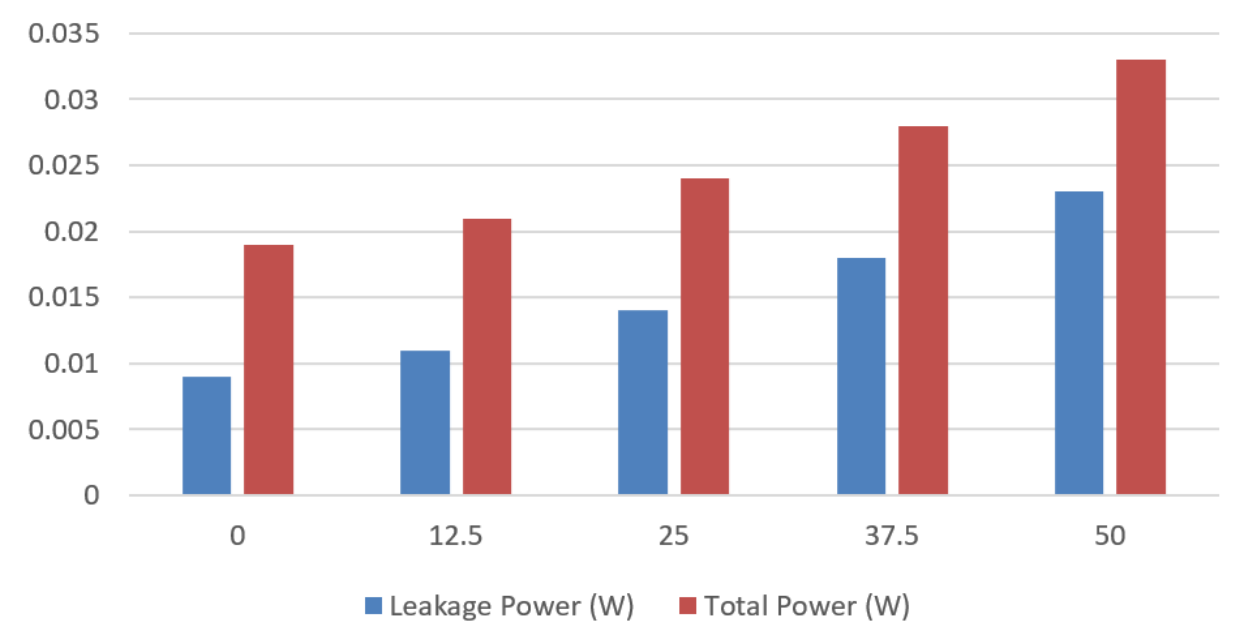

Figure 6. Power Dissipation by Sinusoidal PWM Waveform Generator 
Table. 3 Power Dissipation when Period of Clock is 14 ns and Wave Pulse is 21 ns

\begin{tabular}{|l|l|l|l|l|l|l|}
\hline $\begin{array}{l}\text { Ambient } \\
\text { Temperature } \\
\text { (in C) }\end{array}$ & $\begin{array}{l}\text { Clocks } \\
\text { Power } \\
(\mathrm{W})\end{array}$ & $\begin{array}{l}\text { Logic } \\
\text { Power } \\
(\mathrm{W})\end{array}$ & $\begin{array}{l}\text { Signals } \\
\text { Power } \\
(\mathrm{W})\end{array}$ & $\begin{array}{l}\text { IOs } \\
\text { Power } \\
(\mathrm{W})\end{array}$ & $\begin{array}{l}\text { Leakage } \\
\text { Power } \\
(\mathrm{W})\end{array}$ & $\begin{array}{l}\text { Total } \\
\text { Power } \\
(\mathrm{W})\end{array}$ \\
\hline 0 & 0.001 & 0.000 & 0.000 & 0.006 & 0.009 & 0.016 \\
\hline 12.5 & 0.001 & 0.000 & 0.000 & 0.006 & 0.011 & 0.018 \\
\hline 25 & 0.001 & 0.000 & 0.000 & 0.006 & 0.014 & 0.021 \\
\hline 37.5 & 0.001 & 0.000 & 0.000 & 0.006 & 0.018 & 0.025 \\
\hline 50 & 0.001 & 0.000 & 0.000 & 0.006 & 0.023 & 0.030 \\
\hline
\end{tabular}

For this case we can see from Table 3 and Figure 7 that there is a fall of $60.86 \%$, $52.17 \%, 39.13 \%$ and $21.73 \%$ in the Leakage Power as the ambient temperature is scaled down from 50 (C) to $0,12.5,25,37.5$ (C) degree Celsius Respectively. The total power goes low by a percentage of $42.22 \%, 36.36 \%, 27.27 \%$, and $15.15 \%$ as the temperature level is scaled down from $50(\mathrm{C})$ to $0,12.5,25,37.5$ (C) respectively. There is no change in Clock Power (W) and IOs Power (W) both remains constant value with a value $0.002 \mathrm{~W}$ and $0.008 \mathrm{~W}$ respectively when ambient temperature is increased from 0 (C) to 50 (C). Also see no Logic Power (W) and Signal Power (W) was consumed by the circuit when the ambient temperature is varied from $0(\mathrm{C})$ to $50(\mathrm{C})$. Figure 7 is displaying leakage and total power for the range of ambient temperature when the clock period is $14 \mathrm{~ns}$ and pulse width is $21 \mathrm{~ns}$.

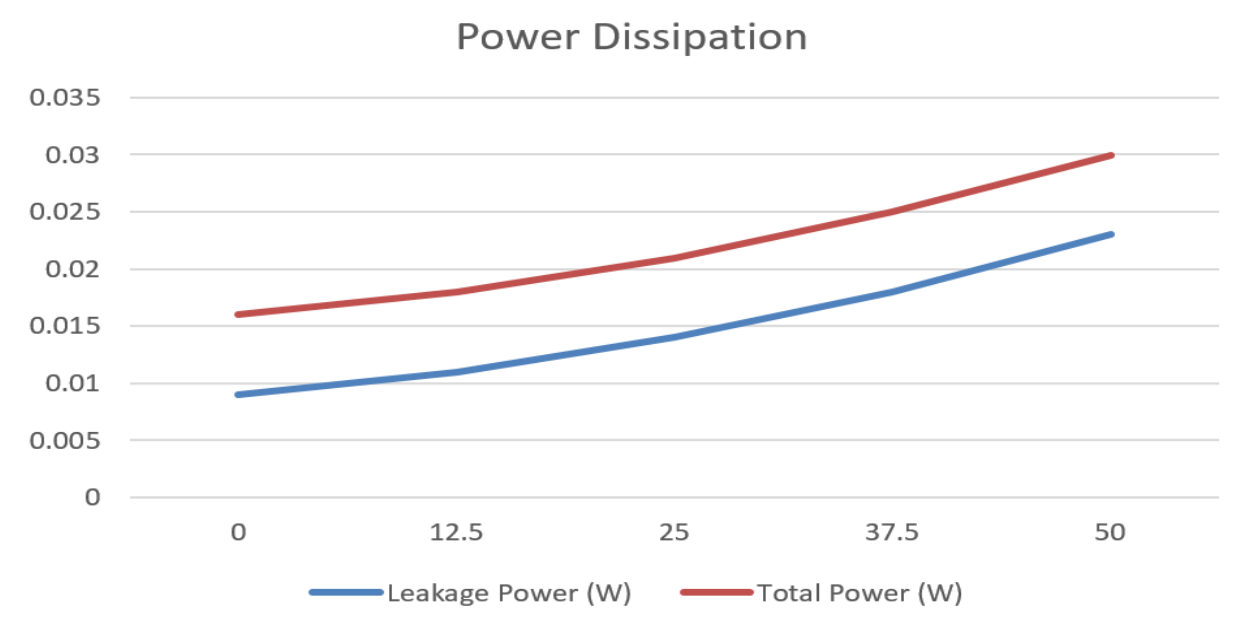

Figure 7. Power Dissipation when Clock Period is 14 ns and Period of Wave Pulse is 21 ns

Table 4. Power Dissipation when Period of Clock is $08 \mathrm{~ns}$ and Wave Pulse is 12 ns

\begin{tabular}{|l|l|l|l|l|l|l|}
\hline $\begin{array}{l}\text { Ambient } \\
\text { Temperature } \\
\text { (in C) }\end{array}$ & $\begin{array}{l}\text { Clocks } \\
\text { Power } \\
(\mathrm{W})\end{array}$ & $\begin{array}{l}\text { Logic } \\
\text { Power } \\
(\mathrm{W})\end{array}$ & $\begin{array}{l}\text { Signals } \\
\text { Power } \\
(\mathrm{W})\end{array}$ & $\begin{array}{l}\text { IOs } \\
\text { Power } \\
(\mathrm{W})\end{array}$ & $\begin{array}{l}\text { Leakage } \\
\text { Power } \\
(\mathrm{W})\end{array}$ & $\begin{array}{l}\text { Total } \\
\text { Power } \\
(\mathrm{W})\end{array}$ \\
\hline 0 & 0.003 & 0.000 & 0.000 & 0.014 & 0.009 & 0.026 \\
\hline 12.5 & 0.003 & 0.000 & 0.000 & 0.014 & 0.011 & 0.028 \\
\hline 25 & 0.003 & 0.000 & 0.000 & 0.014 & 0.014 & 0.031 \\
\hline 37.5 & 0.003 & 0.000 & 0.000 & 0.014 & 0.018 & 0.035 \\
\hline 50 & 0.003 & 0.000 & 0.000 & 0.014 & 0.023 & 0.040 \\
\hline
\end{tabular}


Total power decreases by a percentage of $35 \%, 30 \%, 22.5 \%$, and $12.5 \%$ as the temperature level is scaled down from 50 (C) to $0,12.5,25,37.5$ (C) respectively as shown in Table 4. There is no change in Clock Power (W) that stays at a constant value $0.003 \mathrm{~W}$ and it is the case with IOs Power (W) with a constant value $0.014 \mathrm{~W}$ during the scaling range. It should also be noted that no Logic Power (W) and Signal Power (W) was consumed by circuits when increasing in Ambient temperature from 0 (C) to 50 (C). Figure 8 is displaying leakage and total power for the range of ambient temperature when the clock period is $08 \mathrm{~ns}$ and pulse width is $12 \mathrm{~ns}$.

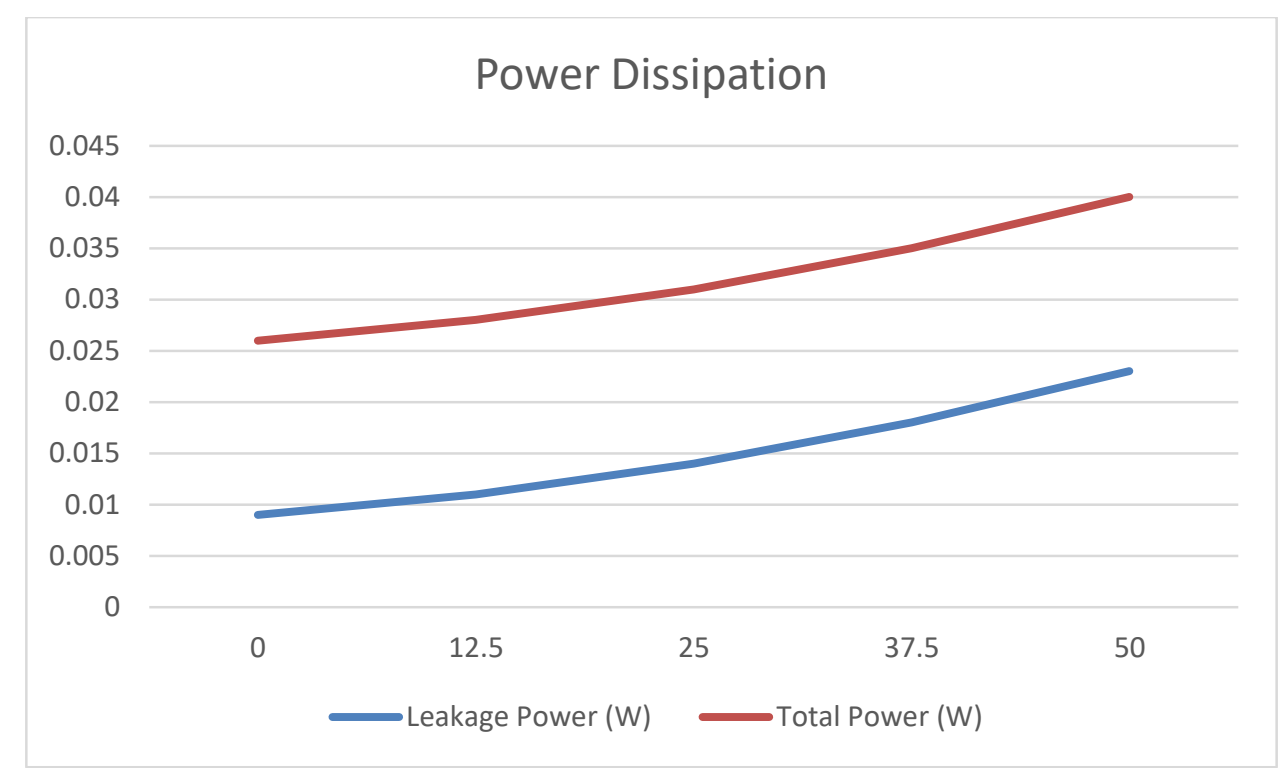

Figure 8. Power Dissipation by Sinusoidal PWM Waveform Generator

Table 5. Power Dissipation when Clock Period is 02 ns and Period of Wave Pulse is 03 ns

\begin{tabular}{|l|l|l|l|l|l|l|}
\hline $\begin{array}{l}\text { Ambient } \\
\begin{array}{l}\text { Temperature } \\
\text { (in C) }\end{array}\end{array}$ & $\begin{array}{l}\text { Clocks } \\
\text { Power } \\
(\mathrm{W})\end{array}$ & $\begin{array}{l}\text { Logic } \\
\text { Power } \\
(\mathrm{W})\end{array}$ & $\begin{array}{l}\text { Signals } \\
\text { Power } \\
(\mathrm{W})\end{array}$ & $\begin{array}{l}\text { IOs } \\
\text { Power } \\
(\mathrm{W})\end{array}$ & $\begin{array}{l}\text { Leakage } \\
\text { Power } \\
(\mathrm{W})\end{array}$ & $\begin{array}{l}\text { Total } \\
\text { Power } \\
(\mathrm{W})\end{array}$ \\
\hline 0 & 0.009 & 0.001 & 0.000 & 0.057 & 0.009 & 0.077 \\
\hline 12.5 & 0.009 & 0.001 & 0.000 & 0.057 & 0.011 & 0.079 \\
\hline 25 & 0.009 & 0.001 & 0.000 & 0.057 & 0.014 & 0.082 \\
\hline 37.5 & 0.009 & 0.001 & 0.000 & 0.057 & 0.018 & 0.086 \\
\hline 50 & 0.009 & 0.001 & 0.000 & 0.057 & 0.024 & 0.092 \\
\hline
\end{tabular}

We can see from Table 5 and Figure 9 there is a fall of $62.5 \%, 54.16 \%, 41.66 \%$ and $25 \%$ in the Leakage Power as the ambient temperature is scaled down from 50 (C) to 0 , 12.5, 25, 37.5 (C) degree Celsius Respectively. The total power goes low by a percentage of $16.3 \%, 14.13 \%, 10.86 \%$, and $6.52 \%$ as the temperature level is scaled down from 50 (C) to $0,12.5,25,37.5$ (C) respectively. There is no change in Clock Power (W) and IOs Power(W) both stays at a value of $0.009 \mathrm{~W}$ and $0.057 \mathrm{~W}$ during ambient temperature scaling from $0(\mathrm{C})$ to $50(\mathrm{C})$. It should also be noted that there is no change in Logic Power (W) which has constant value $0.001 \mathrm{~W}$ and no Signal Power (W) is consumed by the circuit when ambient temperature is scaled from $0(\mathrm{C})$ to $50(\mathrm{C})$. 


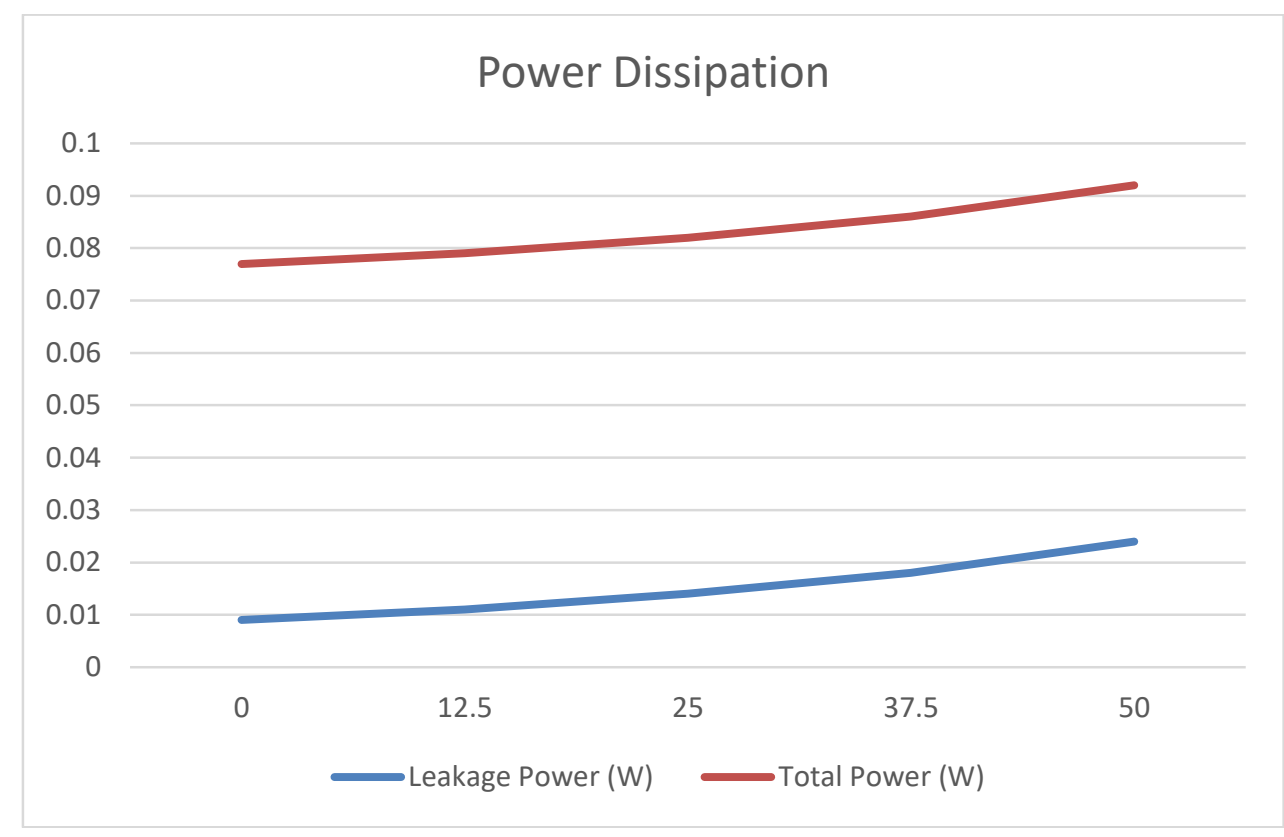

Figure 9. Power Dissipation Clock Period is 02 ns and Period of Wave Pulse is $03 \mathrm{~ns}$

\section{Conclusion}

Reductions in clock, logic, IOs, leakage and total power values are obtained in our simulation. It is obvious from these tables that at higher value of ambient temperature more power is consumed and vice versa. Through this investigation we are able to design a low power sinusoidal PWM waveform generator with an efficient output. Spartan-6 gives low power readings and so is efficient for designing of not only sinusoidal PWM waveform generator but various other electronic designs. The frequency range used from $50 \mathrm{M} \mathrm{Hz}$ to $500 \mathrm{MHz}$ for clock pulse and $33.33 \mathrm{MHz}$ to $333.33 \mathrm{MHz}$ for wave pulse. For this frequency range the value of clock and IOs power remains the same and logic and signal power is negligible for different levels of ambient temperature scaling however due to leakage there is noticeable difference in total power consumed.

\section{Future Scope}

These results can be used in future for making efficient sinusoidal PWM waveform generator on FPGA. In this investigation work has been done on various frequencies at different levels of ambient temperature. It is important to make this useful device efficient and a lot of work can be extended in this field further. We can also use different FPGA families like automotive Artix-7, automotive coolrunner-2, automotive Spartan, automotive Spartan-3A DSP, automotive Spartan-3A, automotive Spartan-3E, automotive Spartan-6, Spartan-3, Spartan-3E. It is important to make this useful device efficient and a lot of work can be done in this field further. 


\section{References}

[1] T. Kumar, B. Pandey, T. Das and B. S. Chowdhry, "Mobile DDR IO Standard Based High Performance Energy Efficient Portable ALU Design on FPGA", Wireless Personal Communications, An International Journal, vol. 76, Issue 3, (2014), pp. 569-578.

[2] S. Madhok, B. Pandey and A. Kaur, M. H. Minver and D. M. A. Hussain, "HSTL IO Standard Based Energy Efficient Multiplier Design using Nikhilam Navatashcaramam Dashatah on 28nm FPGA", International Journal of Control and Automation, vol. 8, no. 8, (2015), pp. 35-44.

[3] B. Pandey, V. Thind, S. K. Sandhu, T. Walia and S. Sharma, "SSTL Based Power Efficient Implementation of DES Security Algorithm on 28nm FPGA", International Journal of Security and Its Application, vol. 9, no. 7, (2015), pp. 267-274.

[4] D. Singh, K. Garg, R. Singh, B. Pandey, K. Kalia and H. Noori, "Thermal aware Internet of Things Enable Energy Efficient Encoder Design for security on FPGA", International Journal of Security and Its Applications, vol. 9, no. 6, (2015), pp. 271-278.

[5] T. Kumar, "CTHS based energy efficient thermal aware image ALU design on FPGA", Wireless Personal Communications, vol. 85, no. 3, (2015), pp. 671-696.

[6] A. Saxena, "HSTL IO Standards Based Processor Specific Green Counter Design on 90nm FPGA", International Journal of Control and Automation, vol. 9, no. 7, (2016), pp. 331-342.

[7] K. Goswami, B. Pandey, D. M. A. Hussain, T. Kumar and K. Kalia, "Input/Output Buffer Based Vedic Multiplier Design for Thermal Aware Energy Efficient Digital Signal Processing on 28nm FPGA", Indian Journal of Science and Technology, vol. 9, no. 10, (2016).

[8] K. Kalia, B. Pandey, T. Das and D. M. A. Hussain, "SSTL Based Low Power Thermal Efficient WLAN Specific 32bit ALU Design on 28nm FPGA”, Indian Journal of Science and Technology, vol.9, no. 10, (2016).

[9] V. Thind, B. Pandey, K. Kalia, D. M. A. Hussain, T. Das and T. Kumar, "FPGA Based Low Power DES Algorithm Design and Implementation using HTML Technology", International Journal of Software Engineering and Its Applications, vol. 10, no. 6, (2016), pp. 81-92. 